Mitteilungen der Österreichischen Geographischen Gesellschaft,

157. Jg. (Jahresband), Wien 2015, S. 9-12

\title{
Geleitwort des neuen Präsidenten
}

\author{
Helmut WoHLSCHLÄGL, Wien* \\ mit 1 Abb. im Text
}

Ich bin in der letzten Hauptversammlung der Österreichischen Geographischen Gesellschaft (ÖGG) im März 2015 zum neuen Präsidenten der Gesellschaft gewählt worden. Ich habe längere Zeit überlegt, ob ich das freundliche und einstimmige Ersuchen des Vorstands der ÖGG, für die Präsidentschaft zur Verfügung zu stehen, annehmen soll, da ich mich nach langer Tätigkeit am Institut für Geographie und Regionalforschung der Universität Wien gerade im Übertritt in den Ruhestand befinde. Aber ich habe dann doch mit Freude und Ambition im Interesse des Fachs Geographie und der Förderung und Unterstützung unserer bereits 1856 gegründeten Gesellschaft, einer der ältesten wissenschaftlichen Gesellschaften der Welt und der zweitältesten Geographischen Gesellschaft im deutschsprachigen Raum, zugesagt.

Mein besonderer Dank gilt meinem Vorgänger als Präsident der Gesellschaft, Herrn Prof. Dr. Christian STAUDACHER, der die ÖGG während seiner fast drei Funktionsperioden dauernden Tätigkeit umsichtig geführt und durch eine Reihe wichtiger organisatorischer und inhaltlicher Neuerungen auf einen guten Weg gebracht hat. So wurde die Gesellschaft

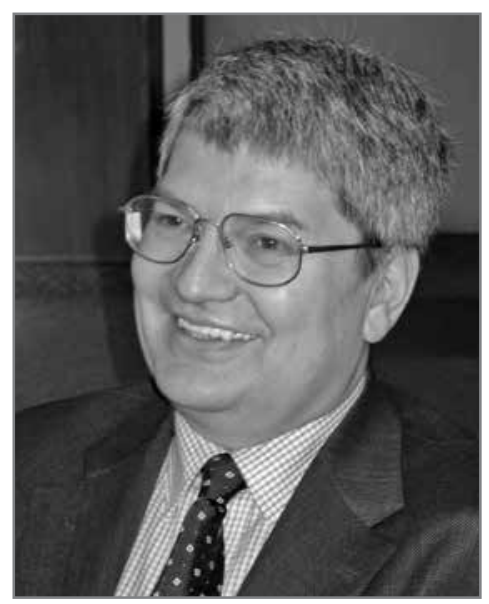

Helmut WoHLSCHLÄGL

\footnotetext{
* Univ.-Prof. Mag. Dr. Helmut WohlschläGl, Präsident der Österreichischen Geographischen Gesellschaft, Institut für Geographie und Regionalforschung der Universität Wien, Universitätsstraße 7/5, A-1010 Wien; E-Mail: helmut.wohlschlaegl@univie.ac.at, http://geographie.univie.ac.at
} 
organisatorisch konsolidiert, die Kanzlei übersiedelt und neu strukturiert, der „Geographie-Fonds“ zur langfristigen Sicherung der Finanzierung der wissenschaftlichen Preise der ÖGG eingerichtet und mit der vierteljährlich erscheinenden Zeitschrift „Geographie aktuell“ ein erfolgreiches Informationsmedium für Mitglieder geschaffen, um nur einige Beispiele zu nennen. Nach nun fast neun Jahren Tätigkeit als Präsident wollte Prof. STAUDACHER diese Funktion nun doch nicht mehr länger ausüben.

Die gute Entwicklung der ÖGG wäre nicht möglich gewesen ohne die engagierte und ehrenamtliche Mitarbeit vieler Mitglieder des Vorstands, insbesondere des Geschäftsführenden Vorstands, denen ich an dieser Stelle ebenfalls sehr herzlich für ihre bisherige Arbeit danken möchte. Es freut mich sehr, dass ein Großteil dieses Teams im Vorstand mit mir weiterarbeiten wird. Dies gilt auch für den Schriftleiter unserer international anerkannten Fachzeitschrift, Herrn Prof. Dr. Peter JoRDAN, dem ich an dieser Stelle für seine langjährige umfangreiche und qualitätsvolle Arbeitsleistung bei der Redaktion und Herausgabe der Mitteilungen der Österreichischen Geographischen Gesellschaft meinen ganz besonderen Dank ausspreche.

Ich möchte an dieser Stelle keine ausführlichen Absichtserklärungen über künftige Entwicklungen der Gesellschaft abgeben, einige Aspekte meiner künftigen Arbeit erscheinen mir aber doch wichtig: So will ich die Kommunikation mit den Mitgliedern unserer Gesellschaft verstärken und mich in diesem Zusammenhang generell bemühen, das Leistungsangebot der ÖGG für ihre Mitglieder zu intensivieren. Großen Wert möchte ich auch auf die Pflege einer engen organisatorischen und fachinhaltlichen Zusammenarbeit mit den Zweigvereinen und Zweigstellen der ÖGG in den Bundesländern sowie mit den Fachgruppen unserer Gesellschaft legen, da diese ein zentraler und tragender Teil der ÖGG sind, der wichtige Impulse zur Weiterentwicklung unserer Gesellschaft einbringt. Ich halte auch Kooperationen mit anderen wissenschaftlichen Gesellschaften und Institutionen im Inland wie im Ausland im Interesse der ,Sichtbarkeit‘ der Geographie und der ÖGG für wichtig und möchte diese gern verstärken.

Ein weiterer bedeutsamer Aspekt ist die Mitgliederwerbung: Zahlreiche Mitglieder halten unserer Gesellschaft schon seit vielen Jahren die Treue, wofür ich mich sehr herzlich bedanke. Wir alle sollten aber auch versuchen, verstärkt den Nachwuchs in unserem Fach, seien es nun Lehrkräfte an den Schulen, wissenschaftliche Mitarbeiter und Studierende an den Universitäten und Pädagogischen Hochschulen oder Absolventen, die mittlerweile in einer Vielzahl von Berufsfeldern in der Praxis tätig sind, für die ÖGG zu interessieren - als Mitglied oder gar durch Mitarbeit innerhalb der Gesellschaft. Vor diesem Hintergrund möchte ich die ÖGG auch gern als eine Art Interessenvertretung der Anliegen der Geographie und der Geographen einschließlich ihrer engeren Nachbargebiete (z.B. Angewandte Geographie und Raumforschung, Kartographie und Geoinformation, Fachdidaktik und Schulgeographie) im öffentlichen Diskurs entwickeln und unsere Gesellschaft in diesem Sinne auch stärker nach außen positionieren.

Neben der Förderung der vierteljährlichen Informations- und Kommunikationszeitschrift „Geographie aktuell““ ist mir vor allem die Unterstützung und Fortführung unserer wissenschaftlichen Fachzeitschrift „Mitteilungen der Österreichischen Geographischen 
Gesellschaft" (MÖGG) ganz besonders wichtig, die mit dieser Ausgabe bereits mit ihrem 157. Jahrgang erscheint und eine der wenigen Fachzeitschriften der Geographie im deutschsprachigen Raum ist, die in internationalen wissenschaftlichen Zeitschriftenrankings aufscheint. Wie der letzten Zusammenstellung des Verbandes der Geographen an Deutschen Hochschulen (VGDH) in der Zeitschrift „Rundbrief Geographie“, Heft 250, vom September 2014 zu entnehmen ist, erfüllen im deutschsprachigen Raum derzeit nur sieben Zeitschriften für Geographie und ihre engeren Nachbargebiete wie Kartographie oder Raumforschung die Kriterien des Institute for Scientific Information (ISI). Die Aufnahme in dessen internationales Zeitschriftenranking erfolgt auf der Basis der Zuordnung eines ,Impact-Factors“, der zunehmend als Messlatte für die wissenschaftliche Reputation einer Zeitschrift und ihren wissenschaftlichen Einfluss, gemessen am Ausmaß, zu dem ihre Beiträge von anderen Fachkollegen zitiert werden, herangezogen wird.

Die MÖGG zählen als einzige österreichische Geographie-Fachzeitschrift neben sechs Zeitschriften aus Deutschland zu dieser kleinen Gruppe international besonders angesehener Publikationsorgane unseres Fachs. Das hat auch zur Folge, dass erfreulicherweise die Zahl der zur Veröffentlichung angebotenen Beiträge jedes Jahr jene, die nach einem anonymen doppelten Begutachtungsverfahren dann tatsächlich in den nächsten Band aufgenommen werden können, weit übersteigt. Die MÖGG haben aber nicht nur eine wichtige Funktion als Fachzeitschrift der Geographie im deutschen Sprachraum, sondern genießen auch hohes Ansehen in Ostmittel- und Südosteuropa, wie sich an der starken und zunehmenden Nachfrage in den östlichen und südlichen Nachbarstaaten Österreichs und den zahlreichen zur Veröffentlichung angebotenen Artikeln aus dieser Region zeigt.

Neben einem jährlich wechselnden Themenschwerpunkt, dem drei bis fünf Beiträge gewidmet sind, bieten die MÖGG mit ihren variierenden Beiträgen ein breites Spektrum von Fachaufsätzen zu Grundsatzfragen unseres Faches sowie zur Humangeographie, Physiogeographie, Regionalforschung, Kartographie und Fachdidaktik, in denen aktuelle Forschungsergebnisse vorgestellt oder theoretische Konzeptionen zu spezifischen Fragestellungen thematisiert werden. Die MÖGG sind aber nicht nur eine rein wissenschaftliche Fachzeitschrift, sondern auch eine ,Gesellschafts-Zeitschrift', denn sie bieten darüber hinaus in jedem Band auch Kurzberichte und kleine Mitteilungen, in denen über wichtige Tagungen oder den Abschluss umfangreicher Projekte berichtet wird. Sie bringen ferner aktuelle Würdigungen und Biographien wichtiger Persönlichkeiten unseres Faches unter besonderer Berücksichtigung von Mitgliedern der ÖGG, einen umfangreichen Teil mit Buchbesprechungen neu erschienener Werke und in der Rubrik „Gesellschaftsnachrichten“ einen jährlichen Überblick über die Entwicklung und die aktuellen Aktivitäten der ÖGG.

In diesem Sinne wünsche ich Ihnen viel Freude mit dem hier vorliegenden neuen Band der MÖGG und hoffe, dass er bei der Vielfalt seiner Inhalte auch für Sie den einen oder anderen interessanten Beitrag und manche wertvolle Information bietet. Helfen Sie mit, die MÖGG auch weiterhin als das führende internationale Fachorgan der wissenschaftlichen Geographie Österreichs zu erhalten und auszubauen - das wäre meine Bitte. 
Wenn Sie finden, dass es sich um überlegenswerte Aspekte handelt, die ich in diesem kurzen Geleitwort dargelegt habe, wenn Sie mit mir Kontakt aufnehmen wollen oder Fragen und Kommentare zu meinen obigen Ausführungen und Anregungen zur ÖGG im Allgemeinen haben oder vielleicht gar in der ÖGG mitarbeiten wollen, dann wenden Sie sich bitte an mich. Ich halte eine aktive Kommunikation zwischen Mitgliedern und Leitung der Gesellschaft sowie einen laufenden Gedankenaustausch für sehr wichtig. 